\title{
Induction of Primordial Germ Cells from Pluripotent Epiblast
}

\author{
Ying Ying ${ }^{1}$, Xiaoxia Qi, and Guang-Quan Zhao* \\ Cecil $H$. and Ida Green Center for Reproductive Biology Sciences, Department of \\ Pharmacology, University of Texas Southwestern Medical Center, Dallas, TX 75390-9051; \\ ${ }^{1}$ Current address: Division of Reproductive Endocrinology and Infertility, Women and \\ Infants Hospital of Rhode Island, Providence, RI 02905
}

Received October 17, 2001; Revised January 29, 2002; Accepted February 1, 2002; Published March 26, 2002

The formation of germ cells during embryogenesis bears the ultimate importance for the continuation of every species. It becomes evident that mechanisms governing germ cell fate specification are not well conserved across the animal kingdom. In most of the invertebrate and nonmammalian vertebrate species, certain maternally derived factors are key to the establishment of germ cell lineage. In contrast, mouse primordial germ cells (PGCs) are induced from the pluripotent epiblast cells before and during gastrulation by the extraembryonic cell-derived signals. The molecular identity for some of these signals has recently been revealed by genetic and epiblast culture experiments. Both bone morphogenetic proteins 4 (Bmp4) and 8b (Bmp8b) are expressed in the extraembryonic ectoderm and are required for PGC formation. Furthermore, BMP4 or BMP8B alone are unable to induce PGCs from cultured epiblasts, while they can in combination, indicating they signal through separate receptor complexes. In addition, Bmp4 homozygous embryos cannot be induced to form PGCs by the synergistic action of BMP4 and BMP8B, suggesting that BMP4 proteins produced by pregastrula embryos are required for epiblast cells to maintain pluripotency. Moreover, $\mathbf{B m p 2}$, a close relative of $\mathrm{Bmp4}$, is expressed in visceral endoderm at the time of PGC specification, and inactivation of Bmp2 results in a reduction in PGC number, revealing a novel function of visceral endoderm in PGC generation in the mouse.

KEY WORDS: mammals, mouse, gastrulation, epiblast, germ cells, germ cell development, PGC (primordial germ cell), totipotency, pluripotency, BMP (bone morphogenetic protein), BMP2, BMP4, BMP8B, BMP receptors, SMAD1, SMAD5

DOMAINS: gene expression, genetics (mouse), signaling, cell and tissue culture, differentiation and determination, intracellular communication, development 


\section{INTRODUCTION}

During sexual reproduction, the union of a sperm cell and a mature oocyte leads to the formation of a zygote that contains all necessary genetic materials from both parents. In nearly all species, the establishment of germ cell lineage is the most important event and usually occurs at early stages of embryonic development. A significant amount of data has been accumulated in germ cell fate specification in the invertebrate species, such as the Drosophila and Caenorhabditis elegans, through extensive genetic studies. Recent studies on PGC formation in mice with embryological and genetic approaches have greatly increased our understanding of germ cell fate determination in mammals. However, it becomes evident that mechanisms governing germ cell fate specification are not well conserved across the phylogeny. The pathways governing PGC generation in different species are significantly different, even among the vertebrate species[1,2,3,4,5,6,7,8]. In C. elegans, Drosophila, fish, most amphibians, and avian species, it appears that asymmetric distribution of maternally derived factors before gastrulation play crucial roles in the establishment of germ cell lineage. However, in urodeles and mammals, PGCs are induced from pluripotent stem cells during gastrulation by signals derived from the extraembryonic cells. In this review, we will focus on our contemporary understanding of the generation of PGCs in mammals, using the mouse as a model system.

\section{GERM CELL FORMATION IN THE MOUSE}

Between 16-24 h following sperm penetration, the fertilized mouse egg begins to cleave to give rise to blastomeres. Up to 8-cell stage, all blastomeres are considered equally totipotent since each is capable of generating all cell types including trophoectoderm. However, at 16-cell stage, only cells at the center retain pluripotency to form inner cell mass (ICM) and cells at the periphery either lose pluripotency or gain potency to eventually become trophoectoderm at the blastocyst stage (32 cells at embryonic day 3.5 [E 3.5]). At E 4.5, a third cell type, primitive endoderm, appears on the surface of ICM. Primitive endoderm becomes the extraembryonic parietal and visceral endoderm while ICM becomes the epiblast $[9,10]$. At the beginning of gastrulation (E 6.0-6.5), epiblast is a cup-shaped tissue containing pluripotent cells that can give rise to the whole fetus as well as the extraembryonic mesoderm. On top of the epiblast is the extraembryonic ectoderm that will form the major part of the placenta. It is worth noting that ICM and epiblast cells, or their derivative embryonic stem (ES) cells, are regarded by many scientists as pluripotent since it is believed that these cells cannot give rise to trophoectoderm. PGCs are first recognized as a cluster of cells located in the extraembryonic region of the primitive streak for their expression of membrane-bound alkaline phosphatase (AP) at E 7.257.5[11] (Fig. 1). They subsequently migrate through the base of allantois at E 8.0-8.5, the endoderm of the hindgut at E 8.5-9.5, the mesenchyme of the mesentery at E 9.5-10.5, and finally to the genital ridge by E 10.5 .

During mammalian embryonic development, totitpotency and pluripotency resides within the following cells: fertilized egg or zygote, blastomeres, ICM, and many cells within the epiblast. PGCs in culture can form embryonic germ (EG) cells that share many of the same characteristics as ICM- or epiblast-derived ES cells. These characteristics include the ability to contribute to all three germ layers in embryonic chimeras[12]. Therefore, PGCs, especially the migratory ones, are also regarded as pluripotent by many researchers. This notion is further supported by the embryonic expression of a POU domain- and homeodomain-containing transcription factor, OCT4, in all of the above totipotent and pluripotent cells, including PGCs $[13,14,15,16]$. 


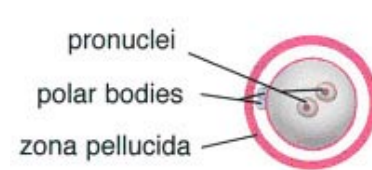

Day 0 fertilized egg

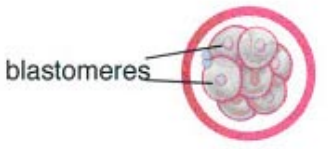

Day 2.5 morula

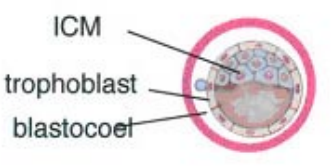

Day 3.5

blastocyst

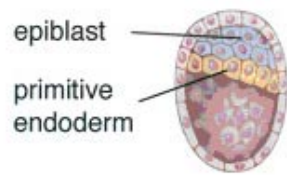

Day 4.5

implanting blastocyst

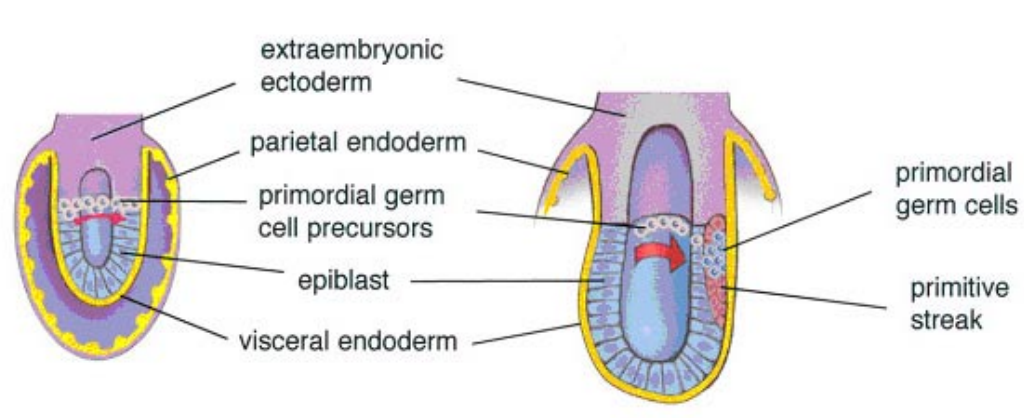

Day 6.0-6.5

pre-spreak stage
Day 7.0-7.25

mid-streak stage

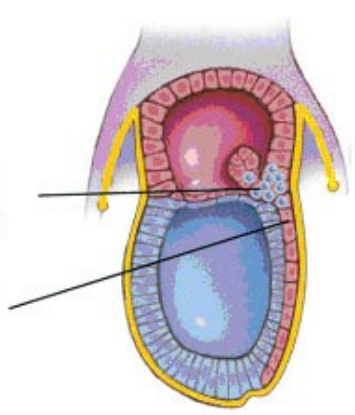

Day 7.5

late streak stage

FIGURE 1. Establishment of germ cell lineage in the mouse. The developmental sequence of a mouse embryo from a fertilized egg (embryonic day 0) to gastrulation, the time of primordial germ cell formation at embryonic day 7.25-7.5 is shown. PGC precursors localized in the proximal epiblast and definitive PGCs in the primitive streak area are depicted as pink and blue circles with darker smaller circles as nuclei, respectively. PGC precursors migrate posteriorly into primitive streak and segregate into PGCs and mesoderm (red cells).

Since strict germ cell lineage or germ cell determinant cannot be detected in preimplantation mouse embryos, efforts have been made to further investigate PGC formation in postimplantation stages. Fate-mapping studies of the mouse embryos at the gastrula stage showed that the precursors of most tissues are derived from certain regions of the epiblast[17,18,19,20]. For example, cells from the proximal epiblast give rise to extraembryonic mesoderm while cells in the distal region of the epiblast will form the neuroectoderm. Lawson and Hage, using fatemapping techniques, found that PGC precursors are located in the proximal epiblast within oneor two-cell diameter of the extraembryonic ectoderm before E 6.5[21]. Importantly, descendants of labeled single cells in the proximal epiblast were found in PGCs and in several other lineages including allantois, yolk sac, and amnion. Therefore, the fate of germ cells is not fixed before gastrulation. Based on the above findings, Tam and Zhou carried out a series of epiblast transplantation experiments in the mouse embryos, including homotypical transplantation (proximal-to-proximal and distal-to-distal epiblasts) and heterotypical transplantation (proximalto-distal and distal-to-proximal epiblasts)[22]. Their results showed that before E 6.5, cells of the distal epiblast (which normally give rise to the neuroectoderm and surface ectoderm) are able to generate PGCs if they are positioned in close proximity with the extraembryonic ectoderm. However, the proximal epiblast cells never form PGCs if they are transplanted into the distal region (far away from the extraembryonic ectoderm). Therefore, before E 6.5, epiblast cells at different locations have the potency to generate PGCs only if they are in close proximity to the extraembryonic ectoderm. These results indicate that the specific local signals emitted by the extraembryonic ectoderm are critical for the induction of PGCs or PGC precursors. This postulate is further supported by the coculture of epiblasts with extraembryonic ectoderm[23]. PGCs are 
formed within the cultured distal epiblast cells only in the presence of the extraembryonic ectoderm, but not if epiblasts are cultured with STO feeder cells alone.

\section{PGC-INDUCING SIGNALS}

The above studies provide solid embryological evidence that PGC formation in the mouse (possibly most mammals) is induced by signals from the extraembryonic ectoderm rather than predetermined by maternal factors. Therefore, the further identification and characterization of such signals should advance our understanding of the mechanisms governing germ cell formation and even the maintenance of totipotency or pluripotency. As shown by Lawson et al. and Ying et al., genes encoding BMP4 and BMP8B, two growth factors of the BMP family, are similarly expressed in the extraembryonic ectoderm at E 5.5-7.5[24,25]. Very importantly, embryos with targeted deletion of Bmp4 never form PGCs, while Bmp8b null mutants have no or very few PGCs. Thus, these two genes are expressed in the right place and time to serve as the extraembryonic ectoderm-derived signals for PGC precursor formation.

BMPs are members of the transforming growth factor $\beta$ (TGF $\beta$ ) superfamily[26,27]. Based on their sequence identity at amino acid level, these proteins are grouped into several classes. The 60A and DPP classes are among the best characterized. The 60A class includes the Drosophila $60 \mathrm{~A}$ protein and the vertebrate BMP5, BMP6, BMP7, BMP8A, and BMP8B proteins, while the DPP class consists of the Drosophila DPP and the vertebrate BMP2 and BMP4. BMPs are synthesized as precursors with a large $\mathrm{N}$-terminal prodomain and a $\mathrm{C}$-terminal mature domain. The monomers of BMP proproteins dimerize intracellularly through disulfide bonds and the mature region is released as a result of the proteolytic cleavage during secretion. Therefore, BMPs (like other TGF $\beta$ superfamily members) function as homodimers or heterodimers to elicit their biological activities in cell proliferation, cell survival, differentiation, and cell fate determination[26]. For example, in Drosophila, the maternal DPP is required for dorsal-ventral patterning at blastoderm stage, while the zygotic expression is required for the development of the heart, midgut, eye, and appendages[28]. Mice deficient in BMP2 or BMP4 die before midgestation due to defects in mesoderm formation and heart development[29,30]. Several members of the 60A class also play important roles during embryonic development and organogenesis. For example, targeted inactivation of $B m p 7$ results in defects in the kidney due to the failed proliferation, survival, and/or differentiation of the nephrogenic mesenchyme, as well as defects in eye and bone formation[31,32].

BMPs trigger cellular responses by binding to heteromeric receptor complexes that contain type I and type II receptor subunits. Both receptor types are serine/threonine protein kinases and both are essential for signal transduction[33,34,35,36]. Type II receptors have constitutively active kinase activity that phosphorylate type I receptors upon ligand and receptor complex formation. The phosphorylated type I receptors relay signals to downstream target proteins. Several putative receptors have been identified for their ability to transduce BMP signals, including type I receptors ALK1, ALK2, ALK3 (BMPR and BMPRIA), and ALK6 (BMPRIB), and type II receptors BMPRII (TALK), ActRIIA, and ActRIIB[37,38,39,40,41,42,43,44,45,46].

Among the type I receptors, Alk3 is the only one clearly shown to be expressed in the epiblast. Alk6 is not expressed until E 9.5 in the mouse embryo, and its mutants do not show obvious germ cell defects, suggesting that it is unlikely for Alk6 to play a role in PGC formation[37,47]. Alk2 is mainly expressed and functions in visceral endoderm during gastrulation[48,49]. The expression of Alk1 during gastrulation has not been reported; inactivation of Alkl resulted in a phenotype similar to that of Tgf $\beta 1$ mutants in angiogenesis, suggesting that ALK1 may serve as the receptor for TGF $\beta$ family[50]. Thus, ALK3 seems to be the best candidate of type I BMP receptor in PGC induction since it is expressed in the epiblast and mutation in Alk3 causes embryonic lethality before mesoderm or PGC formation[46]. All 
three putative type II receptors for BMPs (BmprII, ActrIIa, and ActrIIb) are expressed in the epiblast during gastrulation[51,52]. Mutation in BmprII results in a phenotype very similar to that of Alk3 mutants[51]. Therefore, the possible functions of Alk3, BmprII, ActrIIa, and ActrIIb in PGC formation deserve further investigation.

SMAD proteins have been shown to play pivotal roles in the signal transduction of the TGF $\beta$ superfamily[27,41]. It has also been shown that different SMADs transduce signals for different subfamilies of this superfamily. For example, SMAD1, 5, and 8 transduce signals of BMPs, while SMAD2 and SMAD3 transduce signals of TGF $\beta$ s and activins. Smad1 and Smad5 are expressed in the proximal epiblast cells during gastrulation while $S m a d 8$ is mainly expressed in allantois[53,54,55]. Thus, it is likely that SMAD1 and SMAD5 serve as the signal transducers for BMP4 and BMP8B during PGC induction. This notion is supported by the recent findings that targeted mutations in either of them resulted in the absence of PGCs or a severe reduction PGC number[54,55].

\section{Two BMP Pathways are Required and Sufficient for PGC Induction from Cultured Epiblasts}

Bmp4 and Bmp $8 b$ are expressed in the extraembryonic ectoderm, and both are required for PGC generation in the mouse. In order to investigate why two similar genes are required for the same PGC induction process, Ying et al.[25] first examined whether one gene is required for the expression of the other. Their in situ hybridization experiments did not reveal a significant change of Bmp8b expression in Bmp4 homozygous mutant embryos at E 6.5 in comparison with that of the wild-type embyos, and the same was true for Bmp4 expression among Bmp $8 b$ mutants. Thus, it is unlikely that one is required for the expression of the other[25].

Bmp4 heterozygotes show a 50\% reduction in PGC number compared with wild-type embryos on several genetic backgrounds as a result of the haploid insufficiency[24]. Any further compromise in BMP4 signaling pathway in these heterozygotes, including reduction in ligands, receptors, or SMADs, should exacerbate the phenotype of PGC defects in these mutants. Therefore, if BMP4 and BMP8B function similarly or equally through the same receptor complexes, mutation in Bmp $8 b$ should exacerbate the mutant phenotype of Bmp 4 heterozygotes. As shown by Ying et al., there is no obvious difference in PGC numbers between Bmp4+/mutants and Bmp4+/-; Bmp $8 b+/-$ double mutants[25]. These results show that BMP4 and BMP8B proteins do not have an additive effect in PGC induction and further point to two possible scenarios. First, BMP4 and BMP8B function as homodimers through separate receptor complexes. Second, it has been shown that the heterodimers of DPP and 60A classes are much more potent in mesoderm and bone induction[56,57,58]. Thus, BMP4/BMP8B heterodimers may be the true functional ligands in the process of PGC induction.

To distinguish between the above possibilities, Ying et al. established an in vitro system to coculture the epiblasts of E 6.0-6.25 mouse embryos with COS cells producing BMP4 and/or BMP8B proteins[59]. Their results showed that PGCs can be induced from the cultured epiblasts by the COS cells producing both BMP4 and BMP8B, but not by COS cells producing BMP4 or BMP8B alone. Furthermore, similar PGC induction results were obtained with COS cells expressing both BMPs (homodimers and possible heterodimers produced) and with a mixture of equal numbers of BMP4-producing COS cells and BMP8B-producing COS cells (only homodimers produced). These data clearly indicate that BMP4 and BMP8B homodimers are not only required, but their combination is sufficient to induce PGCs from the cultured epiblasts. It is also indicated that these two proteins must signal through separate receptor complexes. Moreover, BMP4 and BMP8B homodimers act directly on the epiblast cells since PGCs can be induced from the isolated epiblast masses in the absence of the visceral endoderm. 


\section{Two Sources of Inductive Signals}

Embryological studies with lineage tracing and epiblast transplantation support a model of PGC induction from the proximal epiblast cells by the extraembryonic ectoderm-derived signals[21,22]. Results from in vivo genetic studies and in vitro epiblast cultures have validated this model and have further revealed that BMP4 and BMP8B are not only required but also sufficient for PGC induction in the mouse[24,25,59]. During and before gastrulation, in addition to the extraembryonic ectoderm, the visceral endoderm is in close contact with epiblast as well. Knowing that visceral endoderm expresses BMP2[60], a very close relative of BMP4, Ying and Zhao tested whether BMP2 participates in PGC induction[62]. Their data revealed that Bmp2 is expressed at detectable levels in the visceral endoderm after E 6.0, especially around the boundary region between the epiblast and the extraembryonic ectoderm. The levels of Bmp2 expression appear to increase as the embryo develops from E 6.0 to E 7.0. Furthermore, a gradient is formed with the highest levels at the posterior and the lowest levels at the anterior. Significantly, Bmp2 homozygous mutants show a 50\% reduction in PGC number attributable to defects in PGC formation. Therefore, a role of visceral endoderm, as a second source of BMPs, in PGC formation has been established during mouse embryogenesis. These genetic studies have also revealed that mutation in Bmp2 exacerbates the PGC defects of Bmp4 heterozygotes; thus, these two genes have an additive effect in PGC induction. However, neither Bmp2 nor Bmp4 has an additive effect with $B m p 8 b$. These data suggest that BMP2 and BMP4 signal directly through the same or similar receptors on the epiblast that are different from those of BMP8B. The biological significance of the anterior-posterior gradient of Bmp2 expression in visceral endoderm is not yet clear. It is possible that such a gradient helps to maintain sufficient levels of BMP2/4 along the migratory path of PGC precursors. Alternatively, BMP2/4 may serve as chemotactic factors to pluripotent stem cells or PGCs precursors; thus, a gradient of BMP2 may guide PGC precursors to the primitive streak. The latter hypothesis is supported by the fact that PGCs are largely trapped in the yolk sac if Bmp4 is not expressed in the extraembryonic mesoderm[61].

\section{Two Steps of Induction}

The combination of genetic studies and in vitro epiblast cultures strongly supports a notion that the DPP class of BMPs and the 60A-member BMP8B signal as homodimers through separate receptor complexes in PGC precursor induction. Therefore, it is anticipated that defects in PGC formation in Bmp8b and Bmp4 null mutants can be rescued by BMP8B and BMP4 proteins. As shown by Ying et al., COS cells expressing BMP8B proteins indeed rescue the PGC defects of $B m p 8 b$ mutants as revealed by AP staining[59]. Surprisingly, similar experiments with COS cells producing BMP4 proteins fail to induce PGCs from Bmp4 null mutants. Moreover, Bmp4 null embryos (E 6.0-6.25) fail to produce any PGCs even in the presence of COS cells producing both BMP4 and BMP8B, although the same COS cells can induce PGCs from isolated wild-type epiblast masses[59]. These results clearly reveal that BMP4 proteins are required prior to E 6.0 for epiblast cells to gain or maintain germline competency before the synergistic action of BMP4 and BMP8B. Bmp4 expression is detected in the inner cell mass at E 3.5, and is then gradually switched to the extraembryonic ectoderm by E 5.5[24,60]. Thus, the epiblast cells and their precursors have already been or are continuously exposed to BMP4 prior to E 6.0. These data indicate that at least two steps of BMP signaling are required to induce PGCs or to maintain the status of pluripotency: (1) BMP4 is required before E 6.0 and (2) BMP2/4 and BMP8B are required after E 6.0 for the establishment of germ cell lineage in the mouse (Fig. 2). 


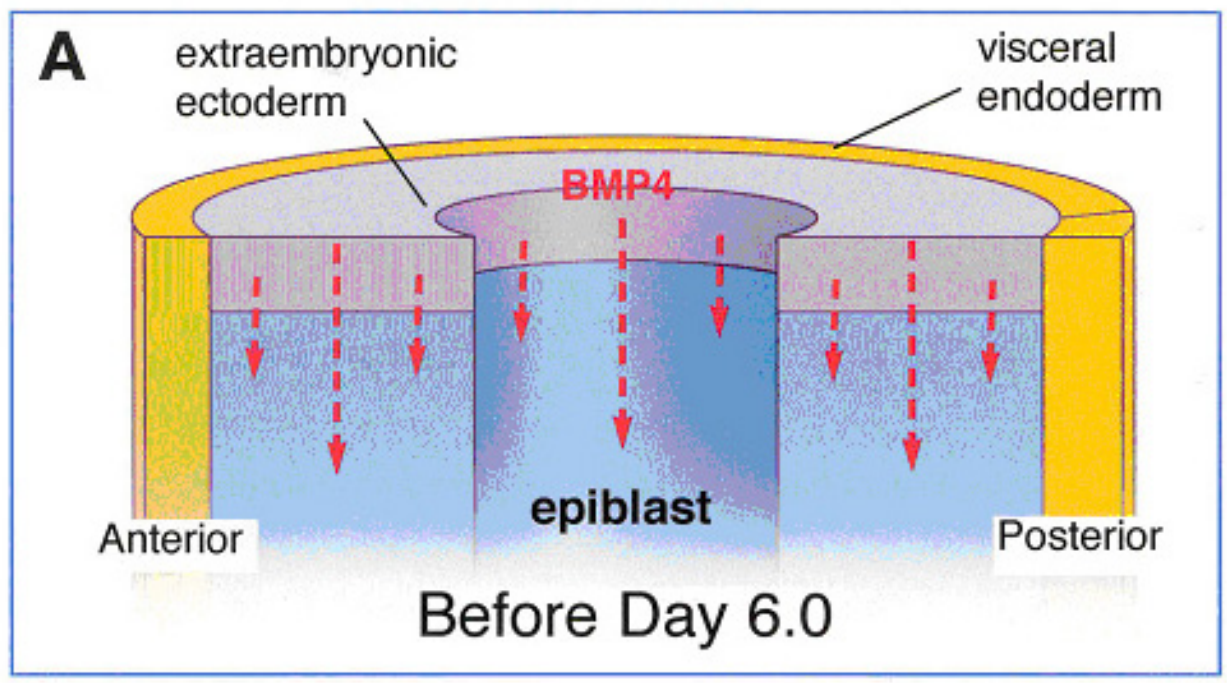

B

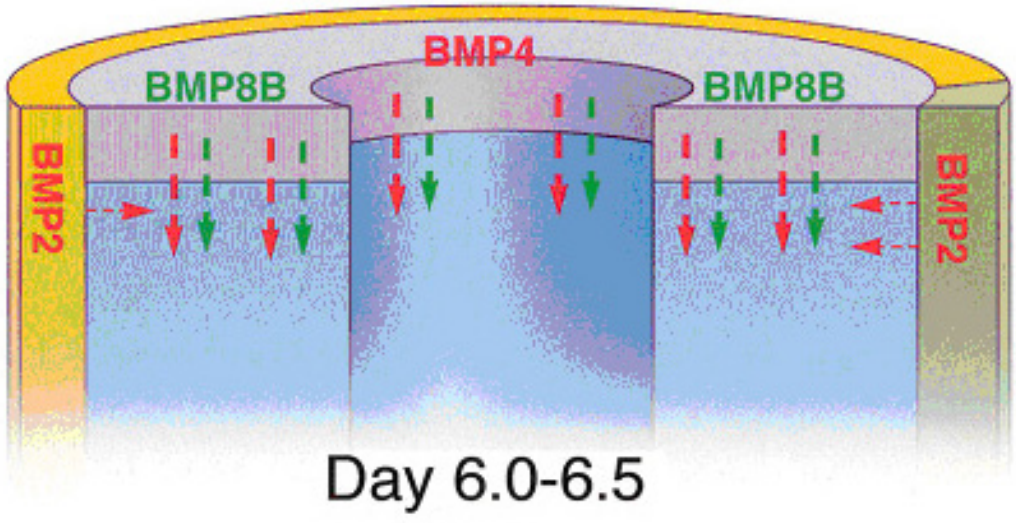

FIGURE 2. Two steps of PGC induction by multiple BMPs during mouse embryogenesis. The diagrams only show one half of the mid portion of a pregastrula embryo. (A) Before E 6.0, BMP4 produced by the extraembryonic ectoderm is required for the epiblast cells to maintain pluripotency or germ cell competency that is essential for subsequent inductions. (B) At E 6.0-6.5, BMP4 and BMP8B homodimers directly bind to separate receptor complexes on cells within the proximal epiblast to synergistically instruct them to develop toward a germ cell fate. In addition, visceral endoderm-derived BMP2 acts in a similar way as BMP4 for germ cell induction. Visceral endoderm at the posterior region expresses higher levels of BMP2 (two small red arrows) than the anterior region (one small red arrow). BMP2 and BMP4 are indicated in red, while BMP8B is depicted in green.

\section{CLOSING REMARKS}

The temporal and spatial expression patterns of Bmp2, Bmp4, Bmp8b, Smad1, Smad5, and their respective mutant phenotypes have established that two BMP signaling pathways (BMP2/4 pathway and BMP8B pathway) are required for PGC formation in the mouse. These two pathways are also sufficient to induce PGCs from cultured epiblasts. Nevertheless, the molecular links (receptor complexes) between BMPs and SMAD proteins remain elusive. It is not clear what receptor subunits participate in BMP2/4 or BMP8B signal pathways and whether these two pathways share common subunits. It also remains to be determined whether BMP2/4 and BMP8B signal through distinct SMAD proteins or converge on both SMAD1 and SMAD5. Furthermore, it is not clear what the target genes are downstream of SMAD1 and SMAD5 in the proximal 
epiblast cells. Finally, since Oct4 is functionally required for pluripotency and consequently for PGC formation, the relationship of BMP signaling and OCT4 activity should also be an important question. It appears that such possible connection between BMPs and OCT4 is further complicated by germ cell nuclear factor (GCNF) that directly suppresses the Oct4 expression[63]. All of these questions will be addressed in the future.

\section{ACKNOWLEDGEMENTS}

This work is supported by grants from the National Institute of Child Health (HD-36218 and HD39154) and the Basil O’Connor Starter Scholar Research Award to G.-Q. Zhao.

\section{REFERENCES}

1. Beams, H.W. and Kessel, R.G. (1974) The problem of germ cell determinants. Int. Rev. Cytol. 39, 413-479.

2. Nieuwkoop, P.D. and Sutasurya, L.A. (1976) Embryological evidence for a possible polyphyletic origin of the recent amphibians. J. Embryol. Exp. Morphol. 35, 159-167.

3. $\quad$ McLaren, A. (1999) Signaling for germ cells. Genes Dev.13, 373-376.

4. Wylie, C. (1999) Germ cells. Cell 96, 165-174.

5. Matova, N. and Cooley, L. (2001) Comparative aspects of animal oogenesis. Dev. Biol. 231, 291320 .

6. Knaut, H., Pelegri, F., Bohmann, K., Schwarz, H., and Nusslein-Volhard, C. (2000) Zebrafish vasa RNA but not its protein is a component of the germ plasm and segregates asymmetrically before germline specification. J. Cell. Biol. 149, 875-888.

7. Wakahara, M. (1996) Primordial germ cell development: is the urodele pattern closer to mammals than to anurans? Int. J. Dev. Biol. 40, 653-659.

8. Starz-Gaiano, M. and Lehmann, R. (2001) Moving towards the next generation, Mech. Dev. 105, 5-18.

9. Hogan, B., Beddington, R., Constantini, F., and Lacy, E. (1994) Manipulating the Mouse Embryo: A Laboratory Manual. Cold Spring Harbor Laboratory Press, Plainview, NY.

10. Beddington, R.S. and Robertson, E.J. (1999) Axis development and early asymmetry in mammals. Cell 96, 195-209.

11. Ginsburg, M., Snow, M.H., and McLaren, A. (1990) Primordial germ cells in the mouse embryo during gastrulation. Development 110, 521-528.

12. Matsui, Y., Zsebo, K., and Hogan, B. L. (1992) Derivation of pluripotential embryonic stem cells from murine primordial germ cells in culture. Cell 70, 841-847.

13. Pesce, M., Wang, X., Wolgemuth, D.J., and Scholer, H. (1998) Differential expression of the Oct-4 transcription factor during mouse germ cell differentiation. Mech. Dev. 71, 89-98.

14. Yeom, Y.I., Fuhrmann, G., Ovitt, C.E., Brehm, A., Ohbo, K., Gross, M., Hubner, K., and Scholer, H.R. (1996) Germline regulatory element of Oct-4 specific for the totipotent cycle of embryonal cells. Development 122, 881-894.

15. Yoshimizu, T., Sugiyama, N., De Felice, M., Yeom, Y.I., Ohbo, K., Masuko, K., Obinata, M., Abe, K., Scholer, H.R., and Matsui, Y. (1999) Germline-specific expression of the Oct-4/green fluorescent protein (GFP) transgene in mice. Dev. Growth Differ. 41, 675-684.

16. Anderson, R., Copeland, T. K., Scholer, H., Heasman, J., and Wylie, C. (2000) The onset of germ cell migration in the mouse embryo. Mech. Dev. 91, 61-68.

17. Lawson, K.A., Meneses, J.J., and Pedersen, R.A. (1991) Clonal analysis of epiblast fate during germ layer formation in the mouse embryo. Development 113, 891-911.

18. Quinlan, G.A., Williams, E.A., Tan, S.S., and Tam, P.P. (1995) Neuroectodermal fate of epiblast cells in the distal region of the mouse egg cylinder: implication for body plan organization during early embryogenesis. Development 121, 87-98.

19. Parameswaran, M. and Tam, P.P. (1995) Regionalisation of cell fate and morphogenetic movement of the mesoderm during mouse gastrulation. Dev. Genet. 17, 16-28.

20. Lawson, K.A. and Pedersen, R.A. (1992) Clonal analysis of cell fate during gastrulation and early neurulation in the mouse. Ciba Found. Symp. 165, 3-21.

21. Lawson, K.A. and Hage, W.J. (1994) Clonal analysis of the origin of primordial germ cells in the mouse. Ciba Found. Symp. 182, 68-84.

22. Tam, P.P. and Zhou, S.X. (1996) The allocation of epiblast cells to ectodermal and germ-line lineages is influenced by the position of the cells in the gastrulating mouse embryo. Dev. Biol. 178, 124-132. 
23. Yoshimizu, T., Obinata, M., and Matsui, Y. (2001) Stage-specific tissue and cell interactions play key roles in mouse germ cell specification. Development 128, 481-490.

24. Lawson, K.A., Dunn, N.R., Roelen, B.A., Zeinstra, L.M., Davis, A.M., Wright, C.V., Korving, J.P., and Hogan, B.L. (1999) Bmp4 is required for the generation of primordial germ cells in the mouse embryo. Genes Dev. 13, 424-436.

25. Ying, Y., Liu, X.M., Marble, A., Lawson, K.A., and Zhao, G.Q. (2000) Requirement of Bmp8b for the generation of primordial germ cells in the mouse. Mol. Endocrinol. 14, 1053-1063.

26. Hogan, B.L. (1996) Bone morphogenetic proteins: multifunctional regulators of vertebrate development. Genes Dev. 10, 1580-1594.

27. Massague, J. and Chen, Y.G. (2000) Controlling TGF-beta signaling. Genes Dev. 14, 627-644.

28. Gelbart, W.M. (1989) The decapentaplegic gene: a TGF-beta homologue controlling pattern formation in Drosophila. Development 107, 65-74.

29. Zhang, H. and Bradley, A. (1996) Mice deficient for BMP2 are nonviable and have defects in amnion/chorion and cardiac development. Development 122, 2977-2986.

30. Winnier, G., Blessing, M., Labosky, P.A., and Hogan, B.L. (1995) Bone morphogenetic protein-4 is required for mesoderm formation and patterning in the mouse. Genes Dev. 9, 2105-2116.

31. Dudley, A.T., Lyons, K.M., and Robertson, E.J. (1995) A requirement for bone morphogenetic protein7 during development of the mammalian kidney and eye. Genes Dev. 9, 2795-2807.

32. Luo, G., Hofmann, C., Bronckers, A.L., Sohocki, M., Bradley, A., and Karsenty, G. (1995) BMP-7 is an inducer of nephrogenesis, and is also required for eye development and skeletal patterning. Genes Dev. 9, 2808-2820.

33. Nellen, D., Affolter, M., and Basler, K. (1994) Receptor serine/threonine kinases implicated in the control of Drosophila body pattern by decapentaplegic. Cell 78, 225-237.

34. Ruberte, E., Marty, T., Nellen, D., Affolter, M., and Basler, K. (1995) An absolute requirement for both the type II and type I receptors, punt and thick veins, for dpp signaling in vivo. Cell 80, 889-897.

35. ten Dijke, P., Miyazono, K., and Heldin, C.H. (1996) Signaling via hetero-oligomeric complexes of type I and type II serine/threonine kinase receptors. Curr. Opin. Cell Biol. 8, 139-145.

36. Weis-Garcia, F. and Massague, J. (1996) Complementation between kinase-defective and activationdefective TGF- beta receptors reveals a novel form of receptor cooperativity essential for signaling. EMBO J. 15, 276-289.

37. Dewulf, N., Verschueren, K., Lonnoy, O., Moren, A., Grimsby, S., Vande Spiegle, K., Miyazono, K., Huylebroeck, D., and Ten Dijke, P. (1995) Distinct spatial and temporal expression patterns of two type I receptors for bone morphogenetic proteins during mouse embryogenesis. Endocrinology 136, 2652-2663.

38. Kawabata, M., Chytil, A., Moses, H.L. (1995) Cloning of a novel type II serine/threonine kinase receptor through interaction with the type I transforming growth factor-beta receptor. J. Biol. Chem. 270, 5625-5630.

39. Liu, F., Ventura, F., Doody, J., and Massague, J. (1995) Human type II receptor for bone morphogenic proteins (BMPs): extension of the two-kinase receptor model to the BMPs. Mol. Cell. Biol. 15, 3479-3486.

40. Suzuki, A., Thies, R.S., Yamaji, N., Song, J.J., and Wozney, J.M. (1994) A truncated bone morphogenetic protein receptor affects dorsal-ventral patterning in the early Xenopus embryo. Proc. Natl. Acad. Sci. U. S. A. 91, 10255-10259.

41. Heldin, C.H., Miyazono, K., and ten Dijke, P. (1997) TGF-beta signalling from cell membrane to nucleus through SMAD proteins. Nature 390, 465-471.

42. Hoodless, P.A., Haerry, T., Abdollah, S., Stapleton, M., O'Connor, M.B., Attisano, L., and Wrana, J.L. (1996) MADR1, a MAD-related protein that functions in BMP2 signaling pathways. Cell 85, 489-500.

43. Yamashita, H., ten Dijke, P., Huylebroeck, D., Sampath, T.K., Andries, M., Smith, J.C., Heldin, C.H., and Miyazono, K. (1995) Osteogenic protein-1 binds to activin type II receptors and induces certain activin-like effects. J. Cell Biol. 130, 217-226.

44. Chen, Y.G. and Massague, J. (1999) Smad1 recognition and activation by the ALK1 group of transforming growth factor-beta family receptors. J. Biol. Chem. 274, 3672-3677.

45. Macias-Silva, M., Hoodless, P.A., Tang, S.J., Buchwald, M., and Wrana, J.L. (1998) Specific activation of Smad1 signaling pathways by the BMP7 type I receptor, ALK2. J. Biol. Chem. 273, 25628-25636.

46. Mishina, Y., Suzuki, A., Ueno, N., and Behringer, R.R. (1995) Bmpr encodes a type I bone morphogenetic protein receptor that is essential for gastrulation during mouse embryogenesis. Genes Dev. 9, 3027-3037.

47. Yi, S.E., Daluiski, A., Pederson, R., Rosen, V., and Lyons K.M. (2000) The type I BMP receptor BMPRIB is required for chondrogenesis in the mouse limb. Development 127, 621-630.

48. Gu, Z., Reynolds, E.M., Song, J., Lei, H., Feijen, A., Yu, L., He, W., MacLaughlin, D.T., van den Eijnden-van Raaij, J., Donahoe, P.K., and Li, E. (1999) The type I serine/threonine kinase receptor ActRIA (ALK2) is required for gastrulation of the mouse embryo. Development 126, 2551-2561. 
49. Mishina, Y., Crombie, R., Bradley, A., and Behringer, R.R. (1999) Multiple roles for activin-like kinase-2 signaling during mouse embryogenesis. Dev. Biol. 213, 314-326.

50. Oh, S.P., Seki, T., Goss, K.A., Imamura, T., Yi, Y., Donahoe, P.K., Li, L., Miyazono, K., ten Dijke, P., Kim, S., and Li, E. (2000) Activin receptor-like kinase 1 modulates transforming growth factor-beta 1 signaling in the regulation of angiogenesis. Proc. Natl. Acad. Sci. U. S. A. 97, 2626-2631.

51. Beppu, H., Kawabata, M., Hamamoto, T., Chytil, A., Minowa, O., Noda, T., and Miyazono, K. (2000) BMP type II receptor is required for gastrulation and early development of mouse embryos. Dev. Biol. 221, 249-258.

52. Manova, K., De Leon, V., Angeles, M., Kalantry, S., Giarre, M., Attisano, L., Wrana, J., and Bachvarova, R.F. (1995) mRNAs for activin receptors II and IIB are expressed in mouse oocytes and in the epiblast of pregastrula and gastrula stage mouse embryos. Mech. Dev. 49, 3-11.

53. Chang, H., Huylebroeck, D., Verschueren, K., Guo, Q., Matzuk, M.M., and Zwijsen, A. (1999) Smad5 knockout mice die at mid-gestation due to multiple embryonic and extraembryonic defects. Development 126, 1631-1642.

54. Tremblay, K.D., Dunn, N.R., and Robertson, E.J. (2001) Mouse embryos lacking Smad1 signals display defects in extra-embryonic tissues and germ cell formation. Development 128, 3609-3621.

55. Chang, H. and Matzuk, M.M. (2001) Smad5 is required for mouse primordial germ cell development. Mech. Dev. 104, 61-67.

56. Aono, A., Hazama, M., Notoya, K., Taketomi, S., Yamasaki, H., Tsukuda, R., Sasaki, S., and Fujisawa, Y. (1995) Potent ectopic bone-inducing activity of bone morphogenetic protein-4/7 heterodimer. Biochem. Biophys. Res. Commun. 210, 670-677.

57. Israel, D.I., Nove, J., Kerns, K.M., Kaufman, R.J., Rosen, V., Cox, K.A., and Wozney, J.M. (1996) Heterodimeric bone morphogenetic proteins show enhanced activity in vitro and in vivo. Growth Factors 13, 291-300.

58. Nishimatsu, S. and Thomsen, G.H. (1998) Ventral mesoderm induction and patterning by bone morphogenetic protein heterodimers in Xenopus embryos. Mech. Dev. 74, 75-88.

59. Ying, Y., Qi, X., and Zhao, G.Q. (2001) Induction of primordial germ cells from murine epiblasts by synergistic action of BMP4 and BMP8B signaling pathways. Proc. Natl. Acad. Sci. U. S. A. 98, 78587862.

60. Coucouvanis, E. and Martin, G.R. (1999) BMP signaling plays a role in visceral endoderm differentiation and cavitation in the early mouse embryo. Development 126, 535-546.

61. Fujiwara, T., Dunn, N.R., and Hogan, B.L. (2001) Bone morphogenetic protein 4 in the extraembryonic mesoderm is required for allantois development and the localization and survival of primordial germ cells in the mouse. Proc. Natl. Acad. Sci. U. S. A. 98, 13739.

62. Ying, Y. and Zhao, G.Q. (2001) Cooperation of endoderm-derived BMP2 and extraembryonic ectoderm- derived BMP4 in primordial germ cell generation in the mouse. Dev. Biol. 232, 484-492.

63. Fuhrmann, G., Chung, A.C., Jackson, K.J., Hummelke, G., Baniahmad, A., Sutter, J., Sylvester, I., Scholer, H.R., and Cooney, A.J. (2001) Mouse germline restriction of Oct4 expression by germ cell nuclear factor, Dev. Cell 1, 377-387.

\section{This article should be referenced as follows:}

Ying, Y., Qi, X., and Zhao, G.-Q. (2002) Induction of primordial germ cells from pluripotent epiblast. In Reviews in Stem and Progenitor Cells. TheScientificWorldJOURNAL 2, 801-810.

\section{Handling Editor:}

Sally A. Moody, Principal Editor for Cell Cycle (fate) — a domain of TheScientificWorldJOURNAL. 

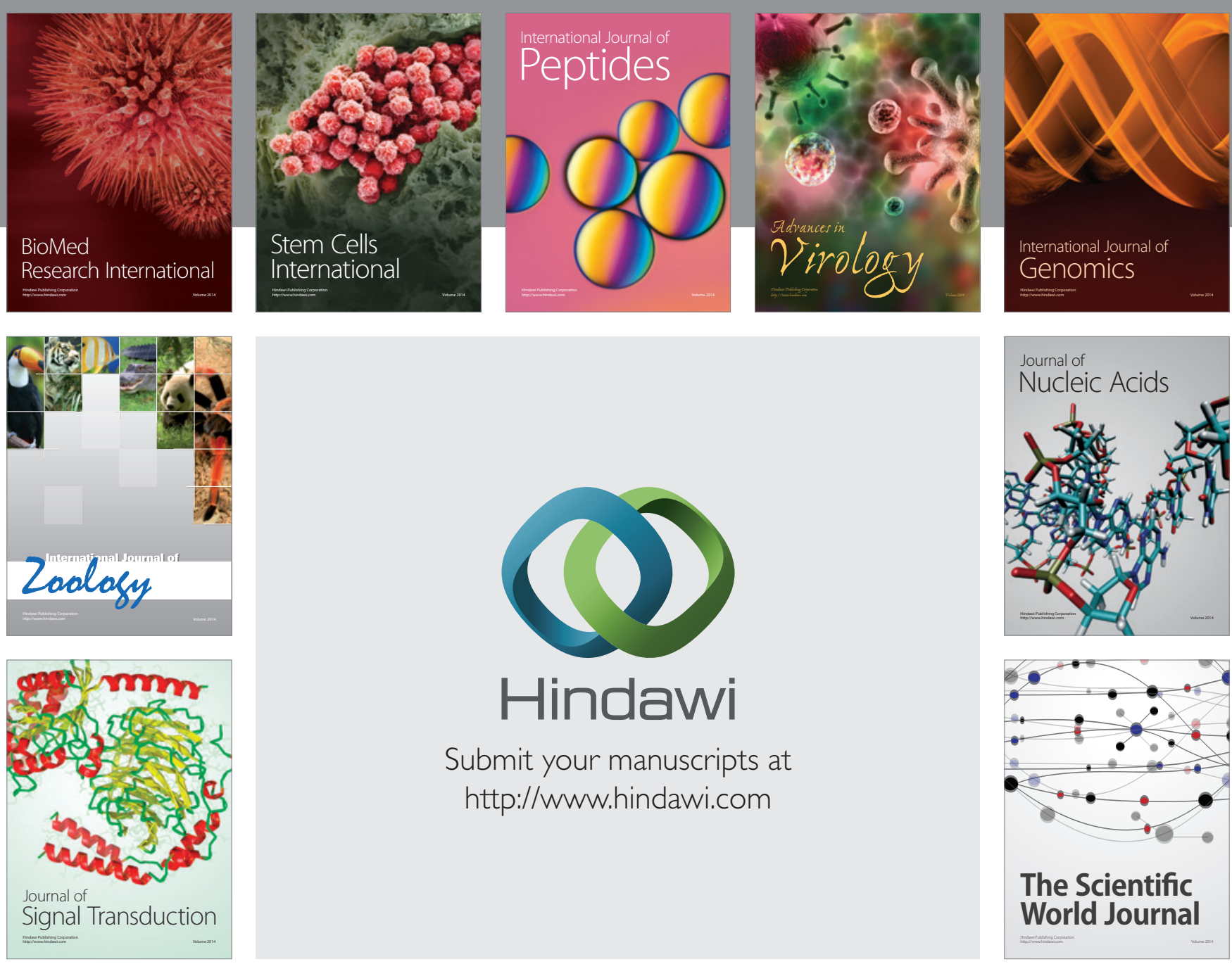

Submit your manuscripts at

http://www.hindawi.com
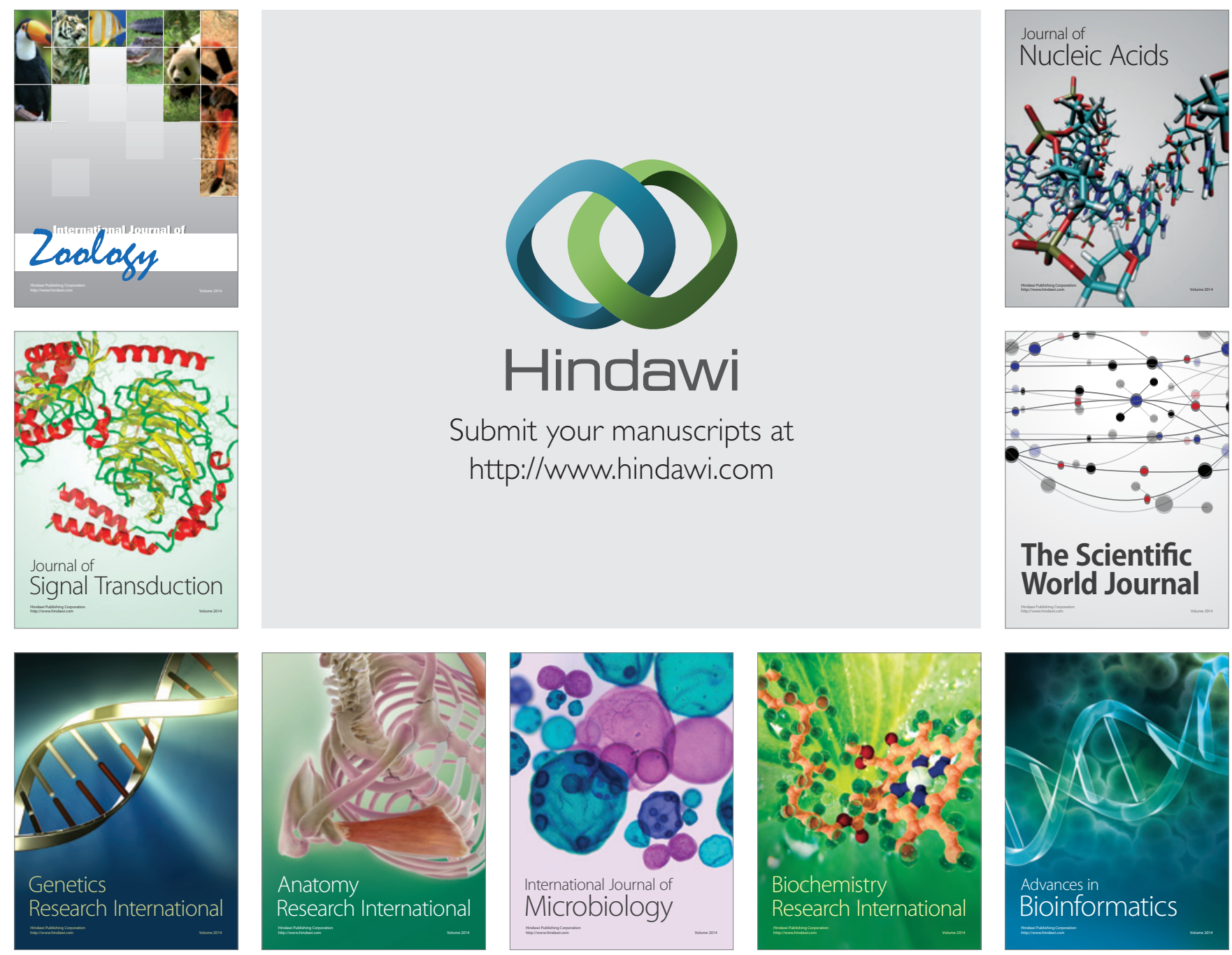

The Scientific World Journal
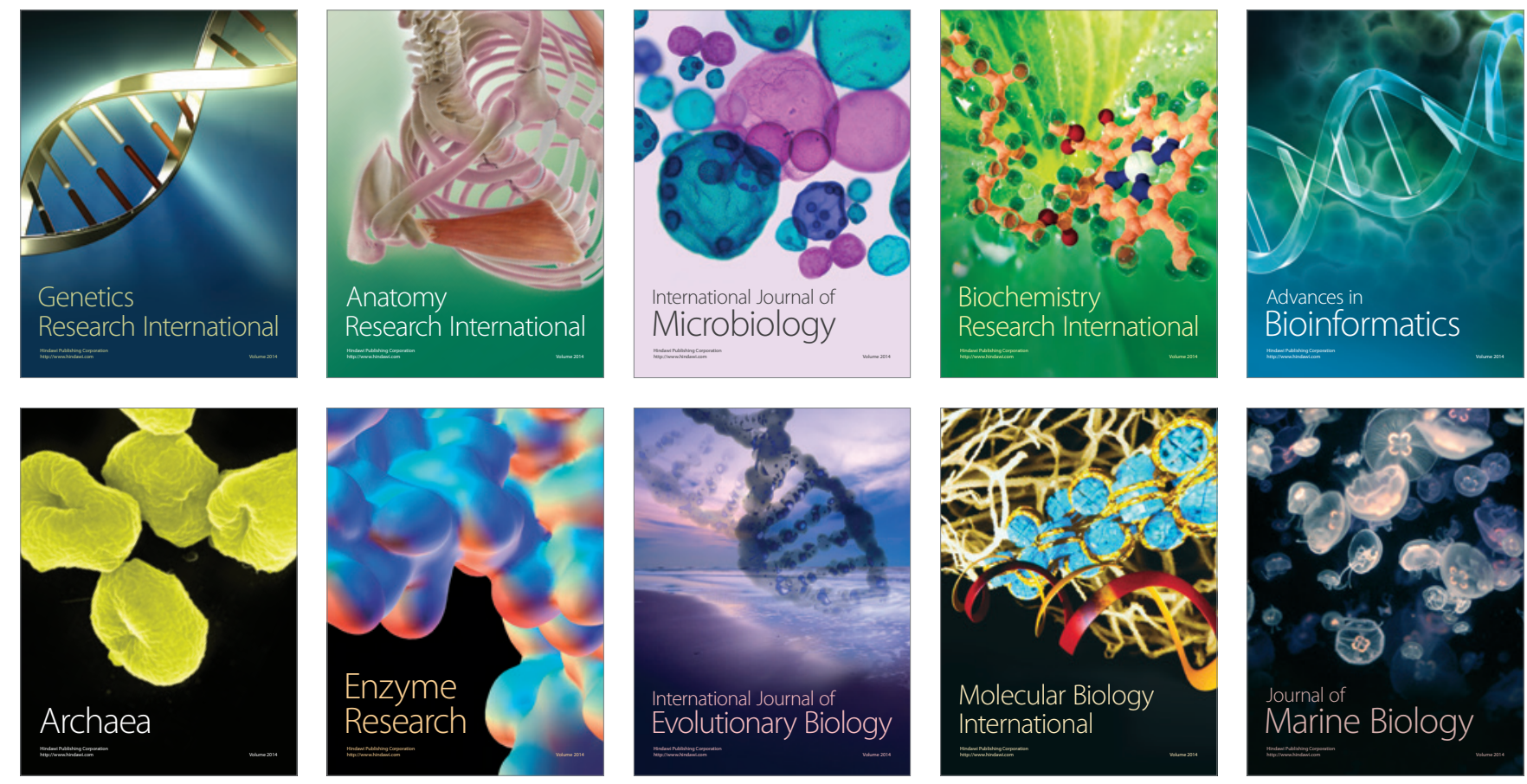